\title{
Porous Biodegradable Polyurethane Nanocomposites: Preparation, Characterization, and Biocompatibility Tests
}

\author{
Regina Coeli Moreira Dias ${ }^{\mathrm{a}}$, Alfredo Miranda Góes ${ }^{\mathrm{b}}$, Rogéria Serakidesc, \\ Eliane Ayres ${ }^{\text {a }}$, Rodrigo Lambert Oréfice ${ }^{\text {a** }}$ \\ ${ }^{a}$ School of Engineering, Department of Metallurgical and Materials Engineering, \\ Federal University of Minas Gerais, Av. Antônio Carlos, 6627, CEP 31270-901 \\ Pampulha, Belo Horizonte, MG, Brazil \\ ${ }^{\mathrm{b}}$ Institute of Biological Sciences, Federal University of Minas Gerais \\ ${ }^{\mathrm{C}}$ Veterinary School, Federal University of Minas Gerais
}

Received: December 2, 2009; Revised: April 29, 2010

\begin{abstract}
A porous biodegradable polyurethane nanocomposite based on poly (caprolactone) (PCL) and nanocomponents derived from montmorillonite (Cloisite ${ }^{\circledast 30 \mathrm{~B}}$ ) was synthesized and tested to produce information regarding its potential use as a scaffold for tissue engineering. Structural and morphological characteristics of this nanocomposite were studied by infrared spectroscopy (FTIR), X-ray diffraction (XRD), small angle X-ray scattering (SAXS) and scanning electron microscopy (SEM). The reaction between polyurethane oligomers with isocyanate endcapped chains and water led to the evolution of $\mathrm{CO}_{2}$, which was responsible for building interconnected pores with sizes ranging from 184 to $387 \mu \mathrm{m}$. An in vitro cell-nanocomposite interaction study was carried out using neonatal rat calvarial osteoblasts. The ability of cells to proliferate and produce an extracellular matrix in contact with the synthesized material was assessed by an MTT assay, a collagen synthesis analysis, and the expression of alkaline phosphatase. In vivo experiments were performed by subcutaneously implanting samples in the dorsum of rats. The implants were removed after 14, 21, and 29 days, and were analyzed by SEM and optical microscopy after tissue processing. Histology crosssections and SEM analyses showed that the cells were able to penetrate into the material and to attach to many location throughout the pore structure. In vitro and in vivo tests demonstrated the feasibility for polyurethane nanocomposites to be used as artificial extracellular matrices onto which cells can attach, grow, and form new tissues.
\end{abstract}

Keywords: polyurethanes, nanocomposites, biocompatibility

\section{Introduction}

A variety of hydrolytically degradable polymers have been developed and tested for tissue engineering scaffold applications ${ }^{1}$. The biodegradable behavior is an advantage for tissue engineering and reconstructive surgery since these materials do not need to be removed and can leave room for tissue regeneration ${ }^{2,3}$. The requirements for these materials to act as a substrate for the attachment and proliferation of different types of cells are not only related to the correct adhesion, proliferation, and preservation of the phenotype, but also to mechanical aspects, given that these substrates should ensure the appropriate performance of the device until the new tissue is capable of restoring the original functionality. Among these polymers, polyurethanes (PU) are of particular interest due to their excellent mechanical properties, which are able to match the mechanical properties of soft tissues ${ }^{4,5}$. These materials consist of alternating hard and soft blocks that can separate in the microphase to form hard and soft domains. The hard segment consists of the diisocyanate and chain extender, whereas the polyester or polyether diol is responsible for the generation of the soft segment.

Hydrolytically unstable polyurethanes are generally achieved by incorporating hydrolysable soft segments, such as poly(caprolactone), into the polymer backbone ${ }^{6,7}$. Hydrolysis of the ester bonds in poly(caprolactone) is known to occur at low rates, particularly in $\mathrm{pHs}$ around $7^{8}$. Higher rates of hydrolysis are observed for poly(caprolactone) with lower molar masses and have been tested in basic and acidic conditions 9 . The rate of hydrolysis of poly(caprolactone) can also be enhanced by the presence and concentration of enzymes, such as lipase ${ }^{10,11}$. Polyurethanes that contain poly(caprolactone) as soft segments commonly present low rates of hydrolysis in neutral $\mathrm{pH}$. These rates are affected by the degree of crystallinity, molar mass of the poly (caprolactone) segment, and the presence of enzymes ${ }^{12,13}$. Chain extension with water is also a wellknown method to obtain polyurethane ureas ${ }^{14}$. During this type of chain extension reaction, carbon dioxide, which can be used to produce a pore structure, is also formed, which is in turn essential for the fast and complete ingrowth of cells. This method of pore generation can avoid the use of potential toxic solvents and porogenic components often used in techniques, such as fiber bolding and salt leaching. A drawback, however, is the reduction in mechanical properties, caused by the presence of the porosity.

Porous polyurethane properties can be improved by introducing nanocomponents derived from clay into the polyurethane matrix to produce nanocomposites. Nanoparticles can also avoid a steep reduction in mechanical properties during biodegradation ${ }^{15}$. Lee et al. ${ }^{16}$ incorporated small amounts of montmorillonite (MMT) into biodegradable poly(l-lactic acid) (PLLA) for the purpose of tailoring the mechanical stiffness of PLLA porous scaffolds.

The possible toxicity of nanoparticles and other nanocomponents that are being considered in new applications derived from the 
nanotechnology approach is recently a matter of much concern ${ }^{17}$. Some results from a series of recent scientific investigations in which cells were cultured in contact with nanoparticles tend to show that carbon nanotubes and metal nanoparticles (such as Ag and Au nanoparticles) might be more toxic than metal oxide nanoparticles (silica, alumina, titanium oxide and zinc oxide) ${ }^{18-23}$. The toxicity seems also to increase with the concentration of the nanoparticles. The level of toxicity may even be useful to kill cancer cells or bacteria if properly controlled ${ }^{19-21}$. Toxicity related to nanoparticles derived from clay minerals is much less known. Some reports have demonstrated that these particles may be considered nontoxic ${ }^{24,25}$, since no histopathological changes could be observed in heart, liver, spleen, lung and kidney of rats after ingestion and intravenous injection of clay nanoparticles, as an indication that they could be eliminated through the kidney system. However, some chemical treatments used to improve the organophilic behavior of the clays may lead to toxicity ${ }^{26}$.

The present study reports on the synthesis of a porous nanocomposites based on hydrolysable polyurethane and MMT nanoparticles used as a three dimensional template for initial cell attachment and subsequent tissue formation. Both in vitro and in vivo tests were performed to verify the possible toxicity and feasibility of using this nanocomposite as a potential matrix for tissue engineering.

\section{Experimental}

\subsection{Materials}

Poly(caprolactone diol) (PCL, $\left.\bar{M}_{\mathrm{n}}=1250 \mathrm{~g} \cdot \mathrm{mol}^{-1}\right)$ and poly(caprolactone diol) $\left(\mathrm{PCL}, \bar{M}_{\mathrm{n}}=2000 \mathrm{~g} \cdot \mathrm{mol}^{-1}\right.$ ) were purchased from Polysciences. Isophorone diisocyanate (IPDI) was provided by Bayer (Brazil). Dibutyl tin dilaurate (DBDLT) was obtained from Miracema Nuodex (Brazil). No chemicals used in this work underwent any form of treatment. The organophilic clay Closite ${ }^{\circledR} 30 \mathrm{~B}$, produced by Southern Clay Products, was used as received.

\subsection{Polyurethane nanocomposite synthesis}

The PU nanocomposite was prepared by the prepolymer mixing process, using a $250 \mathrm{~mL}$ threeneck glass flask equipped with a heating mantel, a mechanical stirrer, and a thermometer within a nitrogen environment. The macrodiol components (PCL 1250, PCL 2000) and Cloisite ${ }^{\circledR} 30 \mathrm{~B}$ were added to the reactor and stirred at $50{ }^{\circ} \mathrm{C}$ to yield a homogeneous mass. IPDI ( $\mathrm{NCO} / \mathrm{OH}$ ratio of 2.0) was then added to the presence of DBDLT, and the reaction was carried out at $70{ }^{\circ} \mathrm{C}$ within a nitrogen environment for 2 hours. The amount of free NCO groups on a percentage basis was determined by the standard dibutyl amine back titration method ${ }^{27}$.

After titration, the amount of deionized water, enough to react with free NCO groups, was added and stirred vigorously for about 10 minutes to ensure that the foaming reaction was completed. The reaction between water and isocyanate endcapped polyurethane oligomers produces urea bonds and $\mathrm{CO}_{2}$ (foaming agent). Urea bonds link polyurethane oligomers to yield high molar mass polymer chains that are responsible for increasing the viscosity of the system. Therefore, during the processing of the reaction, the isocyanate and water, foaming, and an increase in viscosity, all occur simultaneously. The composition of the prepared materials is shown in Table 1.

Table 1. Composition wt.(\%) of the synthesized polyurethane nanocomposite.

\begin{tabular}{ccccc}
\hline PCL & PCL & Cloisite & IPDI $^{\mathrm{a}}$ & Water $^{\mathrm{b}}$ \\
1250 & 2000 & $30 \mathrm{~B}$ & & \\
\hline 28.40 & 45.40 & 4.70 & 20.15 & 1.35 \\
\hline
\end{tabular}

$\mathrm{a}=\mathrm{NCO} / \mathrm{OH}$ molar ratio $=2.0 .(\% \mathrm{NCO}$ free $=4.04) . \mathrm{b}=$ Appropriate amount of water was added to react with isocyanate endcapped polyurethane chains. The amount of catalyst was $0.01 \%$ of DBDLT based on IPDI and PCL.
The porous matrix was produced by casting the viscous polyurethane nanocomposite in a Teflon mold and allowing it to cure in an oven at $60{ }^{\circ} \mathrm{C}$ for 24 hours. Before testing, the porous matrix was also washed with an ethanoldeionized water mixture $(1: 1)$ to remove any impurities created during cutting and manipulation and any eventual residual reagent.

\subsection{Characterization}

FTIR spectroscopy was used to monitor the incorporation of the clay in the polyurethane matrix and to verify the final chemical structure of the polymer. Infrared spectra were collected in a Fourier Transform Infrared Spectrophotometer (FTIR; Perkin Elmer, model Spectrum 1000). Measurements were carried out using the attenuated total reflectance (ATR) technique. Each spectrum was a result of 32 scans with a resolution of $4 \mathrm{~cm}^{-1}$.

An X-ray diffractometer (XRD, Philips model PW 3710, $\lambda=1.54 \AA$ ) was used to evaluate the crystallographic changes in the polyurethane matrix due to the presence of clay. Data were obtained in the $2 \theta$ range of $3.50^{\circ}-89.99^{\circ}$ at a scan rate of $0.01^{\circ} / \mathrm{min}$.

The measurements of Synchrotron Small Angle X-ray Scattering (SAXS) were performed using the beam line of the National Synchrotron Light Laboratory (LNLS, Campinas, Brazil). The photon beam used in the LNLS SAXS beamline comes from one of the 12 bending magnets of the electron storage ring. The white photon beam is extracted from the ring through a highvacuum path. After passing through a thin beryllium window, the beam is monochromatized $(\lambda=1.608 \AA$ ) and horizontally focused by a cylindrically bent and asymmetrically cut silicon single crystal. The focus is located at the detection plane. The X-ray scattering intensity, $\mathrm{I}(\mathrm{q})$, was experimentally determined as a function of the scattering vector " $q$ " whose modulus is given by $q=(4 \pi / \lambda) \sin (\theta))$, where $\lambda$ is the X-ray wavelength and $\theta$ being half the scattering angle.

Each SAXS pattern corresponds to a data collection time of 900 seconds. The parasitic scattering intensity produced by the collimating slits was subtracted from the experimental scattering intensity produced by all the studied samples. All SAXS patterns were corrected to compensate for the nonconstant sensitivity of the detector, for the time varying intensity of the direct synchrotron beam, as well as for differences in sample thickness. Because of the normalization procedure, the SAXS intensity was determined for all samples in the same arbitrary units so that they could be directly compared.

Tensile tests were performed according to the ASTM 638 method by using an EMIC DL 3000 universal testing machine. Nonporous samples were prepared by casting the PU nanocomposite within a closed mold containing venting channels so as to allow $\mathrm{CO}_{2}$, produced by the reaction between water and isocyanate endcapped chains, to be released prior to gelation. Samples were produced in $1 \mathrm{~mm}$ thick sheets that were then cut by ASTM 638 type V dogbone dies.

\subsection{Morphological characterization (SEM)}

Scanning Electron Microscopy (SEM) images were obtained from the cryofracture surface of specimens coated with gold by means of a Jeol JSM 6360 LV SEM apparatus. SEM was performed on porous matrices with and without cells to observe both the structure of the pores as well as other important features, such as the adhesion and morphology of cells. Samples were set in a solution made up of $2.5 \%$ glutaraldehyde, $1 \%$ osmium tetroxide in sodium phosphate buffer, and $\mathrm{pH} 7.4$, and were dehydrated in a graded ethanol series (50-100\%).

\subsection{In vitro cell culture}

Samples of the synthesized nanocomposite after sterilization with ethylene oxide were submitted to in vitro cell culture tests, followed 
by analyses of cell viability and activity by employing an MTT assay (MTT, Chemicon, Temecula, USA) ${ }^{28}$, by determining collagen expression (SIRCOL, Biocolor, Newtonabbey, NI), and by evaluating alkaline phosphatase activity (GIBCO, BRL, NY, USA) ${ }^{29}$. Such tests were performed using primary cultures of neonatal rat calvarial osteoblasts. Briefly, freshly isolated osteoblasts were maintained at $37{ }^{\circ} \mathrm{C}$ in flasks containing $10 \%$ fetal bovine serum (FBS) (Gibco BRL, $\mathrm{NY}, \mathrm{USA}$ ), $5 \% \mathrm{CO}_{2}$, and $95 \%$ air. At confluence, cells were detached by trypsin/collagenase treatment and replated in the wells of a 24-well tissue culture plate (Falcon) with an RPMI culture medium (Sigma, ST. Louis, USA) at a density of $5.10^{4}$ cells/ $\mu \mathrm{L}$. Cells were cultured in contact with nanocomposite rectangular samples $(10 \times 5.0 \times 3.0 \mathrm{~mm})$ for 72 hours. The tests were performed in triplicate and standard tissue culture polystyrene (TCPS) was used as a reference.

Data were analyzed using the student Ttest. Results were considered statistically significant when the $\mathrm{P}$ value was less than $0.05(\mathrm{P}<0.05)$. Results were displayed as the mean \pm standard deviation.

\subsection{In vivo implant}

To study in vivo tissue reactions to the presence of nanocomposites, strips of porous polyurethane nanocomposites, measuring $10 \mathrm{~mm}$ in length, $5 \mathrm{~mm}$ in width, and $3 \mathrm{~mm}$ in thickness, were subcutaneously implanted in the dorsum of rats $(n=3)$. Every strip was implanted via surgical incision under anesthesia and the incisions were closed by sutures. The samples were explanted after 14, 21, and 29 days, and histologically processed. Sections were stained using traditional eosin/hematoxylin protocols and observed through an optical microscope (Axiolab/Carl Zeiss) equipped with a JVC TK C720 V camera. An electron microscope was also used as described above. Tissue regions next to the implant site, but not affected by the implant procedure, were used as positive controls to check and compare the natural morphology of the host tissue with the new tissue generated near the biomaterial. Negative controls were also used. These were obtained by drenching the nanocomposite with toxic chemical groups, which were produced by attacking the polymer with high concentrated sulfuric acid solutions (initially intended to allow the attachment of RGD groups Arg-Gly-Asp).

\section{Results}

\subsection{Characterization of the porous nanocomposite}

The FTIR spectra of the pristine polyurethane and the nanocomposite (Figure 1) confirmed the polyurethane structure with the typical carbonyl absorption band of the PCL ester bond located at $1725 \mathrm{~cm}^{-1}$, and a shoulder at $1696 \mathrm{~cm}^{-1}$, which can be attributed to the urethane and urea carbonyl groups. The absorbance at $3335 \mathrm{~cm}^{-1}$ is consistent with the stretching of the $\mathrm{NH}$ bond and is characteristic of urethane and urea groups. The other characteristic bands are $2900 \mathrm{~cm}^{-1}$ due to the alkane $-\mathrm{CH}$ stretching vibration, $1174 \mathrm{~cm}^{-1}$ due to the coupled $\mathrm{C}-\mathrm{N}$ and $\mathrm{C}-\mathrm{O}$ stretching vibrations, and $1062 \mathrm{~cm}^{-1}$ due to the ester C-O-C symmetric stretching vibration ${ }^{30}$. The absence of the absorbance at $2270 \mathrm{~cm}^{-1}$ indicated a lack of unreacted isocyanate groups consumed during chain extension. The $\mathrm{Si}-\mathrm{O}$ stretching vibration of the clay, located at $1052 \mathrm{~cm}^{-1}$, although overlapping with the abovementioned $1062 \mathrm{~cm}^{-1}$ absorption band, can be noticed in the spectrum of Figure 1b. Most of the observed features in the spectrum of the polyurethane nanocomposite remained the same as those seen in the spectrum of neat polyurethane.

In Figure 2, two broad peaks were detected in the X-ray diffraction curves of the pure polyurethane. This is a typical pattern of materials with a low degree of crystallinity. A different situation can be observed

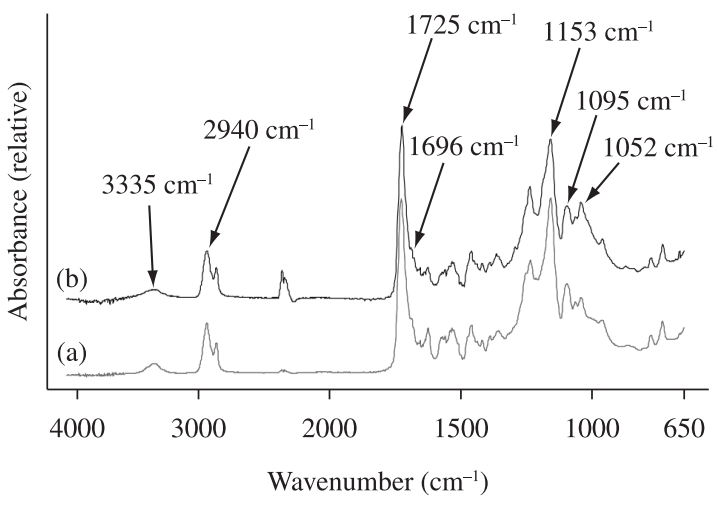

Figure 1. a) FTIR spectra of polyurethane and b) polyurethane nanocomposite.

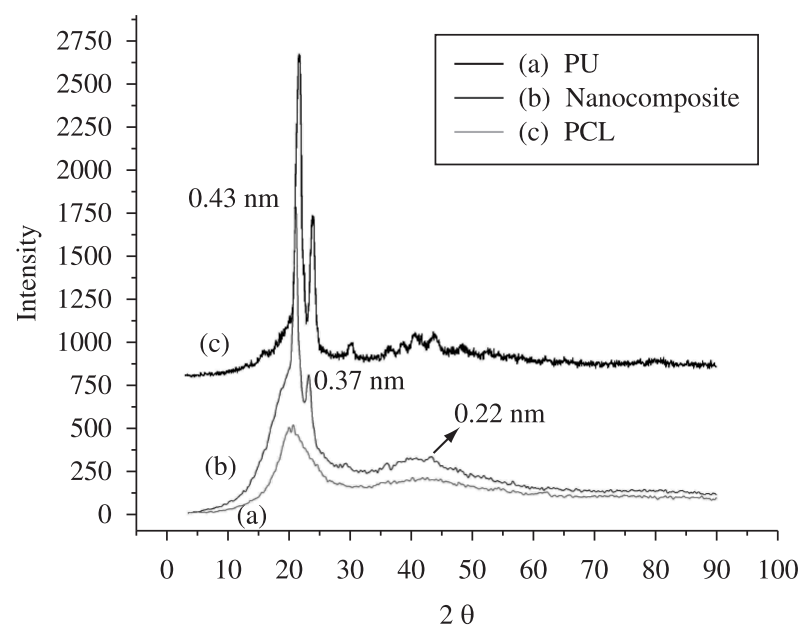

Figure 2. a) X-ray diffraction curves of pure polyurethane, b) polyurethane containing clay particles and c) PCL.

in the XRD pattern of the nanocomposite where sharp peaks are visible, indicating that the clay particles acted as nucleating agents that induced the crystallization of the PCL segments present in the polyurethane, as suggested when comparing the XRD curve of the nanocomposite with the PCL XRD curve (Figure 2c). The (001) plane,

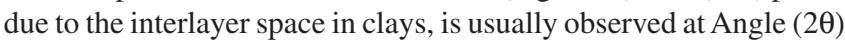
less than $10^{\circ}$. This peak is useful in studying the degree of interaction between the polymer and the nanoparticles, since its shift towards lower angles can be associated with intercalation or even exfoliation (complete delamination of the clay structure) of the polymer within the space between the clay lamellas. However, this reflection is not clearly observed in the XRD curves of Figure 2. Small angle X-ray scattering (SAXS) was thus used to study the nanostructure of the material, since a second phase of colloidal dimensions (such as nanoparticles) dispersed in a matrix can display small angle X-ray scattering if there are differences in electronic densities between the phases. Figure 3 shows SAXS patterns relative to pristine clay and the nanocomposite. Scattering peaks are observed in these curves, which can be converted to distance between phases (L) by using the Bragg's law $\left(\mathrm{L}=2 \pi / \mathrm{q}_{\max }\right.$, where $\mathrm{q}_{\max }$ is the value of $\mathrm{q}$ at the maximum height of the peak). The interlayer distance $((001)$ plane) in pristine clay is close to $1.9 \mathrm{~nm}$, while this and another peak at lower values of q (i.e. 
displaying larger distances between clay nanoparticles) can be seen in the SAXS curve related to the nanocomposite. The presence of these two peaks at the SAXS curve of the nanocomposite indicates that part of the clay became intercalated/exfoliated.

Therefore, the processing of the material succeeded in at least partially producing intercalated/exfoliated nanostructures.

Figure 4 exhibits SEM images of the porous PU nanocomposite to show the presence of an open porosity with pore sizes ranging from 184 to $327 \mu \mathrm{m}$. This type of porosity can then be considered adequate for growing cells.

Tensile properties of dense polyurethane nanocomposite samples are displayed in Table 2 . The values in this table are useful in showing

Table 2. Mechanical properties of polyurethane (PU) and polyurethane having clay nanoparticles.

\begin{tabular}{cccc}
\hline $\begin{array}{c}\text { Samples/ } \\
\text { properties }\end{array}$ & $\begin{array}{c}\text { Elastic } \\
\text { modulus (MPa) }\end{array}$ & $\begin{array}{c}\text { Strength } \\
(\mathrm{MPa})\end{array}$ & $\begin{array}{c}\text { Strain at } \\
\text { failure }(\%)\end{array}$ \\
\hline PU & $21 \pm 4.9 *$ & $50 \pm 2.9$ & $1183 \pm 57$ \\
$\mathrm{PU}+5$ wt. $(\%)$ clay & $206 \pm 14.8$ & $36 \pm 1.5$ & $645 \pm 68$ \\
\hline
\end{tabular}

*Average values \pm standard deviation

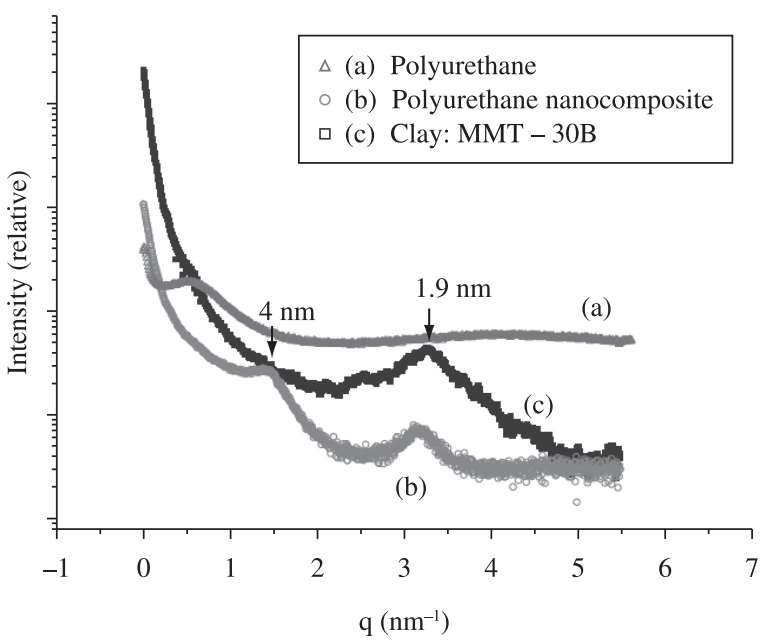

Figure 3. a) SAXS curves of pure polyurethane, b) polyurethane nanocomposite and c) Cloisite ${ }^{\circledR}$ 30B.

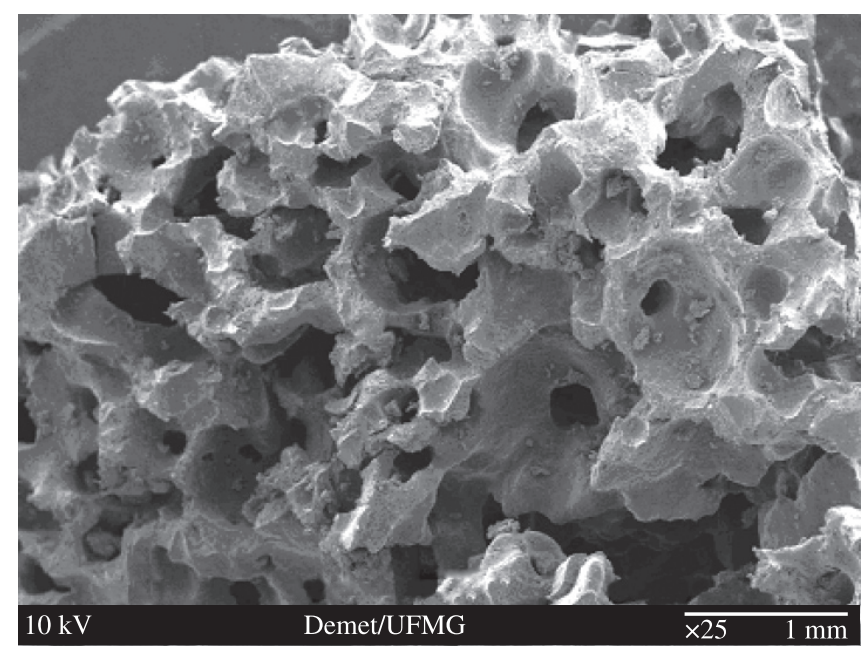

Figure 4. SEM image of the porous polyurethane nanocomposite. the effect of the introduction of clay nanoparticles on the mechanical properties of polyurethanes. It is possible to observe that particularly the elastic modulus of the polymer was highly enhanced when $5 \%$ of the clay nanoparticles were incorporated in the sample. Therefore, the introduction of clay nanoparticles within polyurethanes can alter the mechanical properties in such a way as to produce from soft to stiff materials that can be used to design biomaterials that match the mechanical properties of different types of tissues. Although the results were obtained for nonporous materials, the observed relationship (increase in stiffness, for example) can be extended to porous samples.

\subsection{In vitro test}

Samples were viewed using light microscopy after cell culture. The images presented in Figure 5 show that osteoblastic cells were able to proliferate similarly both in contact with the biomaterial (Figure 5a) and in tissue culture polystyrene (the control Figure 5b).

The MTT assay was used to provide information about cell viability. This method is based on the finding that cells are capable of reducing slightly yellow colored tetrazolium salts to intense red

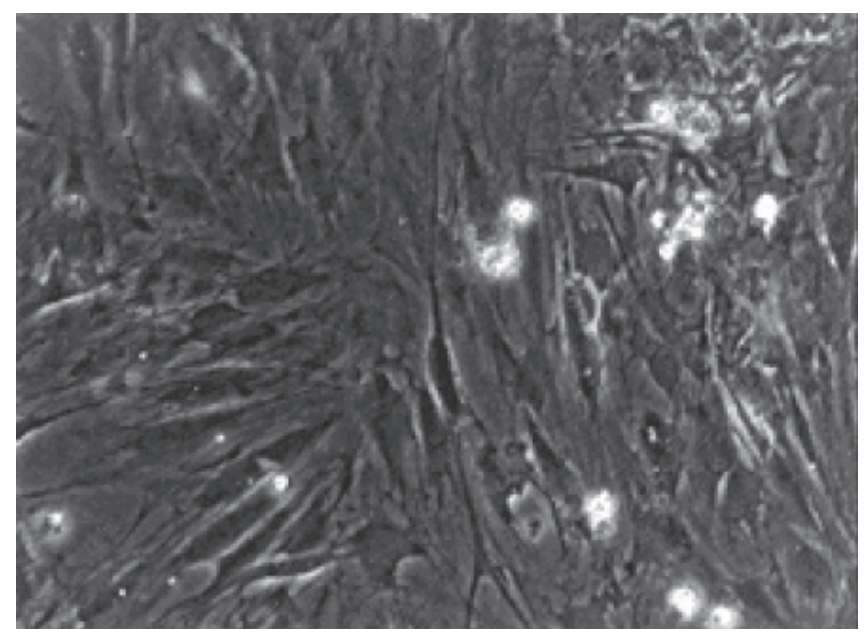

(a)

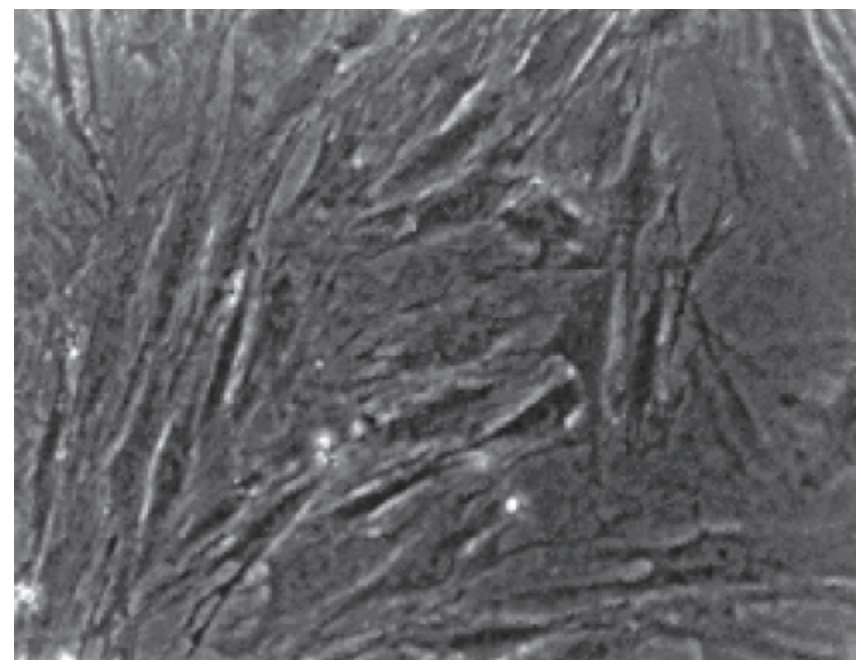

(b)

Figure 5. Osteoblast cell culture: a) cells cultured in contact with the biomaterial; b) cells cultured on tissue culture polystyrene (TCPS - reference). 


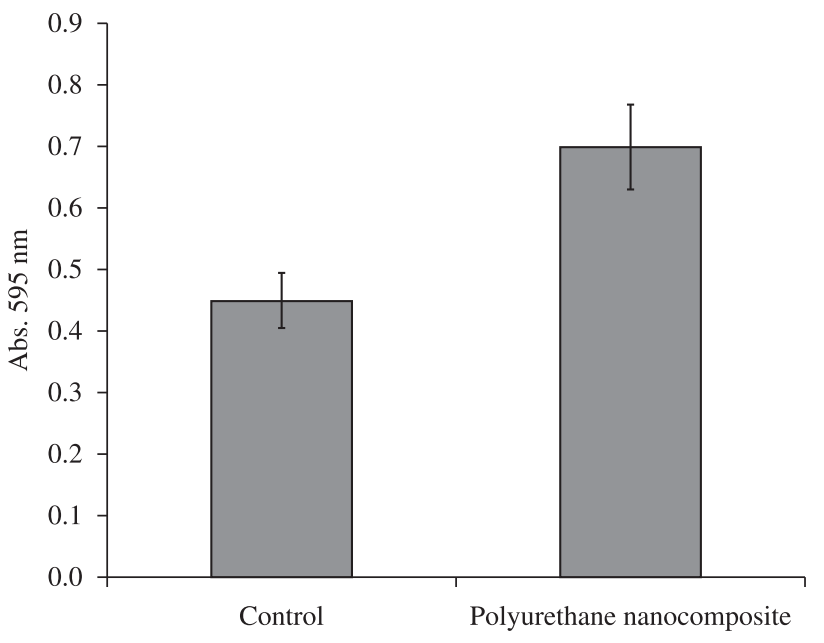

Figure 6. MTT results: osteoblastic cells cultured in contact with PU nanocomposite compared with cells cultured on TCPS (control). The bars indicate standard deviations $(\mathrm{p}<0.05)$.

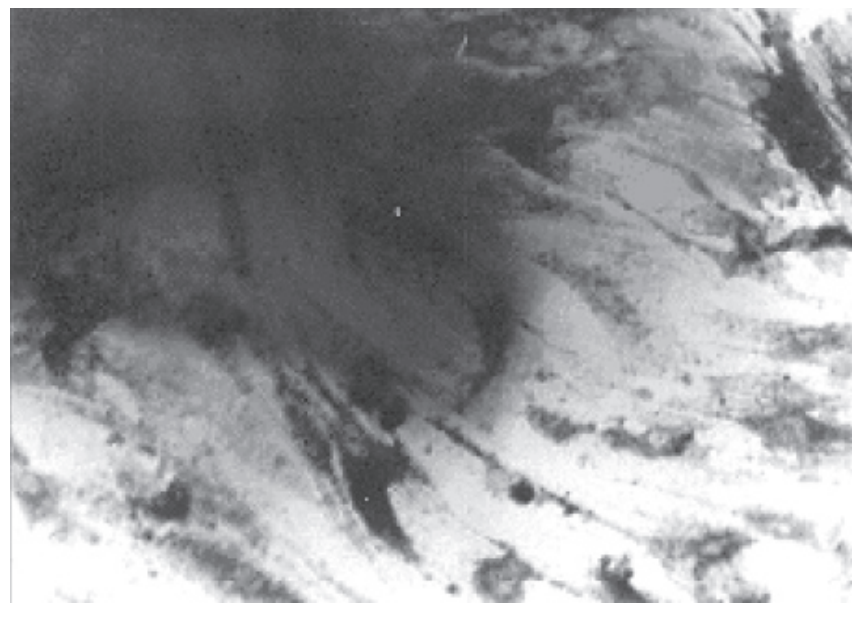

(a)

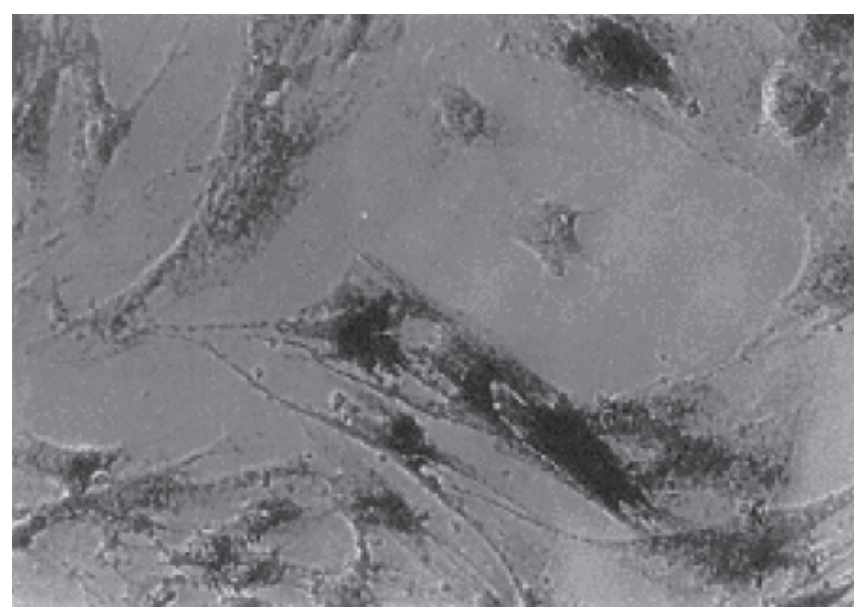

(b)

Figure 7. Osteoblastic cell activity indicated by the production of alkaline phosphatase: purple precipitates indicate the presence of alkaline phosphatase in cultures a) in which cells were cultured in contact with the nanocomposite (darker region: biomaterial) and b) on TCPS with no biomaterial. colored formazan derivatives by an intracellular reduction system mostly located in the mitochondria ${ }^{2}$. After cell lysis and extraction of the formazan crystals from the cell cultures, the MTT absorbance at $595 \mathrm{~nm}$ was measured to provide an indication of osteblastic cell viability. Figure 6 shows that the feasibility of cells cultured in contact with the nanocomposite was higher (33\%) than the feasibility of cells cultured on TCPS. This fact suggests that the cells cultured in contact with the biomaterial showed a higher activity than those cultured on TCPS. This result may well be attributed to the pore structure of the polyurethane nanocomposites, which can accommodate a larger number of active cells within its pore network.

Cells used in this in vitro study were characterized as osteoblasts by detecting the presence of alkaline phosphatase in cell cultures. The presence of alkaline phosphatase is an indicator of osteoblasts. Such a molecule is responsible for the construction of the bone matrix and is used as a marker for cell differentiation in cultures ${ }^{2,31}$. The alkaline phosphatase production was evaluated in this work by means of a BCIP/NBT (5-bromo-4-chloro-3indolyl-phosphate/nitro blue tetrazolium) assay (GIBCO, BRL, NY, USA). This assay is based on a chromagenic reaction initiated by the cleavage of the BCIP phosphate group due to the alkaline phosphatase present in the cells. This reaction produces a proton which reduces NBT to an insoluble purple precipitate. In the light microscopy image of Figure 7a, purple precipitates can be seen around the nanocomposite sample (darker region), as an indication of the presence of the alkaline phosphatase in cultures where cells were cultured in contact with the biomaterial. This result can be compared to a similar result observed when cells were cultured on TCPS (Figure 7b) and proves that osteoblasts were active in both cell cultures.

Osteoblastic activity was also evaluated in terms of the expression of adhesion biomacromolecules, such as collagen type $\mathrm{I}^{3,32}$. Such a biomacromolecule is essential for bone formation and is expressed from early stages of differentiation onwards ${ }^{33}$. The osteoblast collagen production was analyzed by a SIRCOL assay performed on the culture's supernatant. This method is based on the selective binding property of the syriusred dye to the (Gly-X-Y) tripeptide end sequence of mammalian collagen. The solubilized collagen was measured by an optical density analysis at $595 \mathrm{~nm}$. The amount of collagen was calculated based on a linear regression from previously known concentrations of type I collagen and their optical density measurements. Figure 8 shows statistically similar levels of collagen I produced by cells in contact with the polyurethane nanocomposite $(155 \mu \mathrm{g})$ and by cells cultured on TCPS $(175 \mu \mathrm{g})$ (no statistical difference).

\subsection{In vivo tests}

Optical microscopy and SEM analyses of polyurethane nanocomposite samples were performed after 14, 21, and 29 days of implant in the dorsum of rats. Figure $9 \mathrm{a}$ and $\mathrm{b}$ shows a histological evaluation of samples removed after 14 days of implant. The presence of a tissue containing lymphocytes, fibroblasts, endothelial cells, and angiogenic features (formation of new blood vessels) can be observed and indicates the evolution of a typically mild inflammatory reaction. The observed inflammatory reaction at 14 days of implant was not considered severe and was comparable to typical mild foreignbody responses. After 29 days (Figure 10a and b), a reduction in the inflammatory reaction and an accentuated increase in angiogenesis could be observed. No inflammatory cells after 29 days of implant were detected, which indicates no prolonged aggression to the tissue (i.e. low levels of toxicity). In Figure 10c, a tissue area next to the injured area produced by the implant of the biomaterial (but not affected by it) 


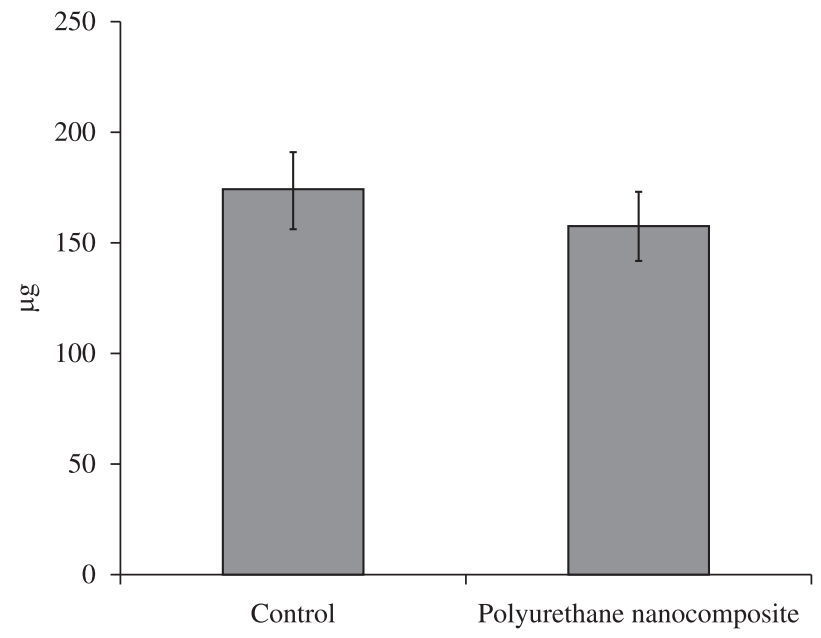

Figure 8. Collagen type I expression: no statistical difference between the expression of collagen by cells cultured in contact with the nanocomposite and on TCPS (control) was noticed.

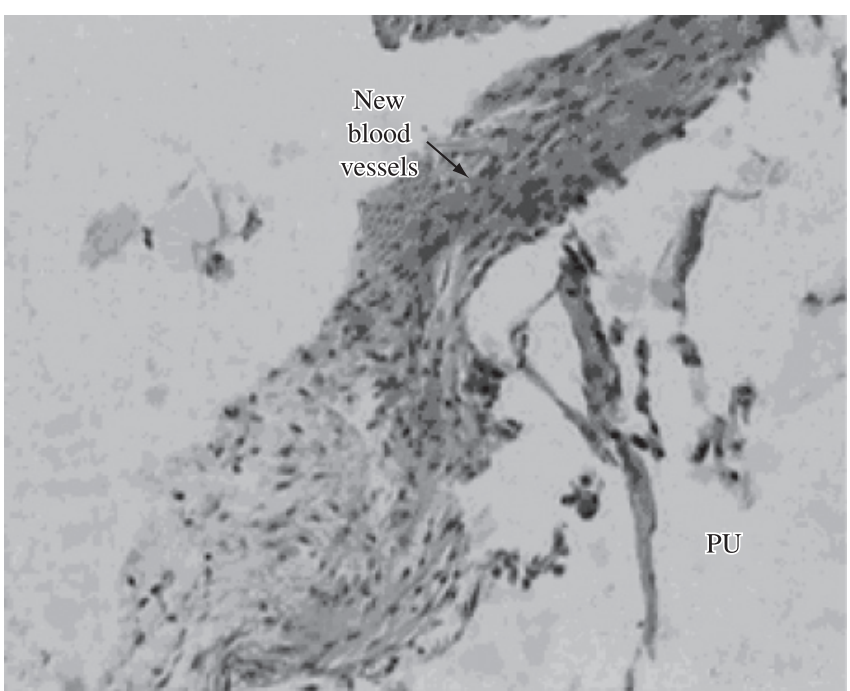

(a)

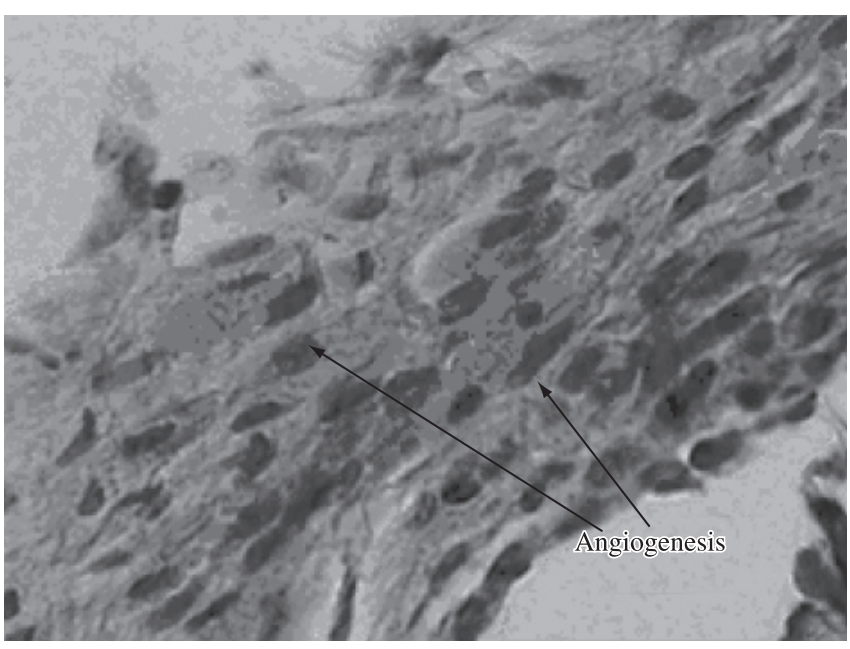

(b)

Figure 9. Optical micrographs of tissues grown close to the surface of the polyurethane nanocomposite. a) Histological evaluation after 14 days (magnification $10 \times$ ) and b) accentuated angiogenesis process (magnification $40 \times$ ) .

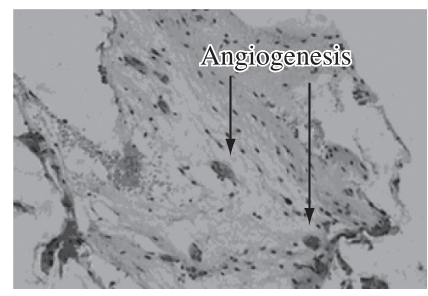

(a)

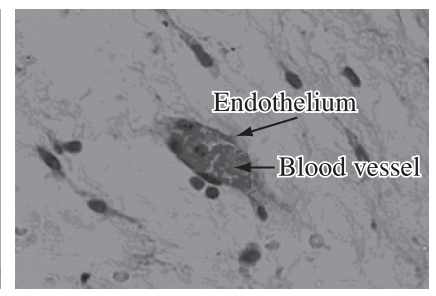

(b)

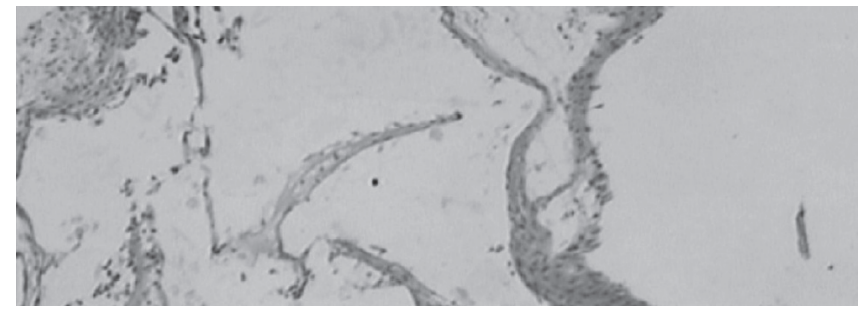

(c)

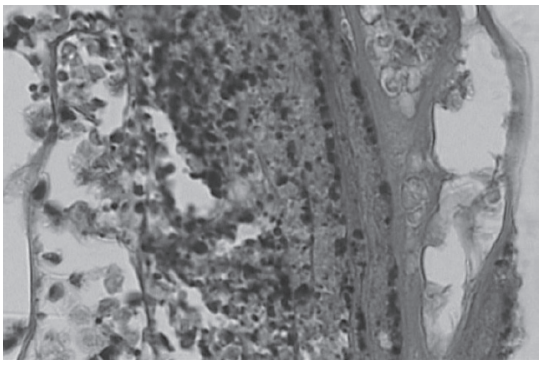

(d)

Figure 10. a) Histological evaluation after 29 days of a polyurethane nanocomposite implant (magnification 20x); b) (magnification 60x); c) positive control (20x); and d) negative control (20x).

can be observed. This positive control is useful when comparing the morphology of the original tissue with the new tissue formed in the presence of the biomaterial. No sign of angiogenesis could be identified in the host tissue (Figure 10c), while new blood vessels could be observed within the implant region, which indicates that the implant of the porous PU nanocomposite was useful in forming new blood vessels within the new tissue.

Figure 10d shows a negative control of the in vivo test. This negative control was obtained by drenching the nanocomposite with toxic chemical groups produced by attacking the polymer with high concentrated sulfuric acid solutions. When implanted, this toxic PU led to the formation of necrotic tissue around the material, indicating high levels of toxicity. The result of this negative control shows that the designated in vivo test was able to detect toxicity associated with biomaterials within 29 days of implant.

Figure 11a and b shows the SEM images of explanted nanocomposite samples after 21 days. It is possible to observe that fibroblasts had colonized the surface of the sample, forming a dense network of connected cells. In addition, the presence of fibers, which may be attributed to the deposition of an extracellular matrix (Figure 11b), could be observed. The crosssection of the material, shown in Figure 11a, demonstrated that cells were able to penetrate and to attach to locations within the pores of the material. 


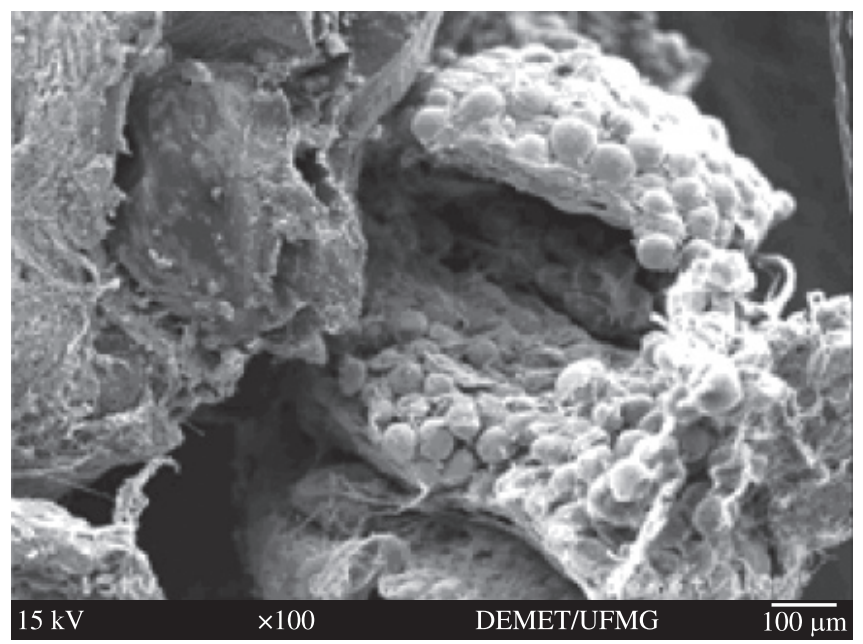

(a)

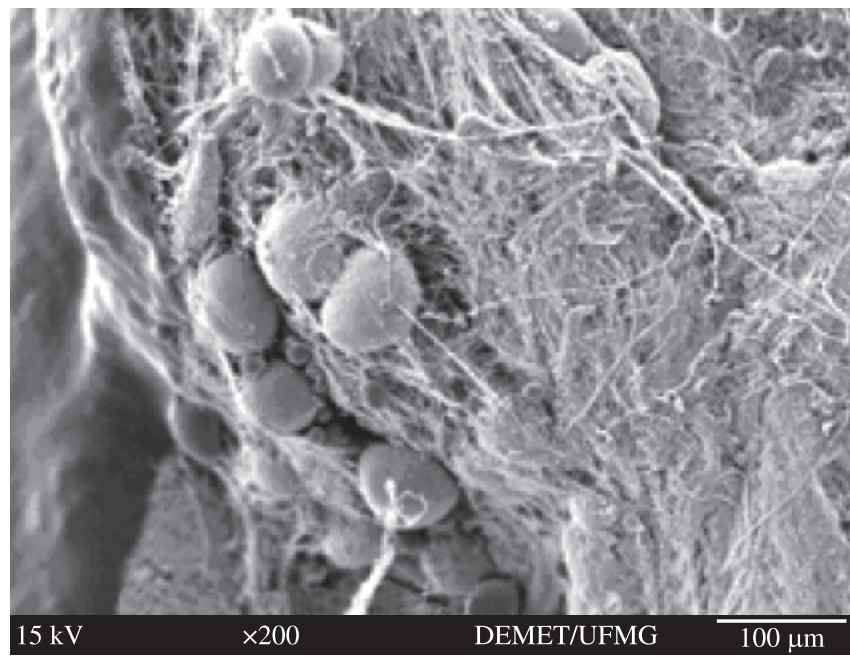

(b)

Figure 11. a) SEM image of the nanocomposite internal pores with colonized cells after 21 days of implant. b) SEM image of fibers attached to polyurethane nanocomposite.

\section{Discussion}

The combination of FTIR, XRD, and SAXS results, shown in Figures 1, 2, and 3, respectively, provided important information regarding the structure of the polyurethane nanocomposite produced in this study. The FTIR spectra in Figure 1 proved that the isocyanate functionality was fully converted into urethane and urea bonds, produced by reactions among the IPDI, polyols, and water. The presence of the clay particles was also detected in the FTIR spectrum of the nanocomposite. XRD and SAXS results were successful in showing that the nanoparticles were at least partially exfoliated within the polymer matrix. The production of this type of dispersion may well be associated with the strong interactions that can occur between the organophilic clay layers that contain $\mathrm{OH}$ groups (Cloisite ${ }^{\circledR}$ 30B is a montmorillonite clay modified with a quaternary ammonium salt that contains both hydrocarbons and $\mathrm{OH}$ groups) and the isocyanate endcapped chains. The presence of an exfoliated (or partially exfoliated) clay dispersed within a polymer is commonly associated with great improvements in properties, such as mechanical properties ${ }^{15}$.
According to Turhani et al..$^{33}$, the degree of interconnection of the pores and pore size, modulate cellular penetration, and proliferation within materials show osteoconductive properties. Pores with diameters of smaller than $10 \mu \mathrm{m}$ inhibit cellular ingrowth, while pore sizes of between 15 and $50 \mu \mathrm{m}$ in diameter aid in fibrovascular colonization. In contrast, pores between 50 and $150 \mu \mathrm{m}$ allow for osteoid growth, and pores larger than $150 \mu \mathrm{m}$ in diameter facilitate internal mineralized bone formation. The pore size distribution and the level of interconnectivity observed in the SEM image in Figure 4 demonstrate that the foaming process derived from the generation of $\mathrm{CO}_{2}$ during the reaction between isocyanate endcapped chains and water was successful in creating a pore structure that is potentially useful to support cell colonization and tissue formation.

In vitro tests showed that the polyurethane nanocomposite obtained in this work is cytocompatible, since no changes in morphology and activity were observed for cells cultured in contact with the biomaterial as compared to cells cultured on TCPS. The use of $\mathrm{CO}_{2}$ as the porogenic agent, rather than organic solvents or salts, is most likely one of the most important aspects that contributes to the lack of cytotoxicity. In vivo results also revealed no tissue toxicity promoted by the nanocomposite within a 29-day period of evaluation. Explanted samples showed that the inflammatory reaction was eliminated after 21 days of implant and that a tissue containing fibroblasts, new blood vessels, and an extracellular matrix was developed. SEM images of the crosssection of the explanted nanocomposite showed that fibroblasts were able to easily penetrate and attach to locations within the pore network and consequently produced pore walls with biocolonized surfaces. Although clay nanoparticles can be considered nontoxic ${ }^{24}$, surfactants used to improve the organophilic behavior of clays were reported to be toxic in some studies ${ }^{26}$. In this work, the synthesis of the nanocomposite was designed to enable the formation of chemical bonds between the alkylammonium (surfactant) and the polymer so as to restrict the free diffusion of this surfactant. The present study employed an alkylammonium which contains $\mathrm{OH}$ groups that were used to react with the isocyanate endcapped polyurethane chains. The formation of urea bonds between the surfactant and the PU may restrict the capability of the species to be easily released. A washing procedure was also used to extract any nonreacted species before performing the biocompatibility tests. The fact that no toxicity could be observed may well prove that these procedures were enough to prevent toxicity.

\section{Conclusions}

Porous biodegradable polyurethane, based on poly(caprolactone) oligomers and reinforced with nanometric clay, was produced by reacting isocyanate endcapped polyurethane chains with water to generate a porogenic and nontoxic $\mathrm{CO}_{2}$ gas. The incorporation of nanoparticles was able to tailor the mechanical properties of the nanocomposite. SEM images showed that the porogenic procedure was able to produce materials containing large (pore sizes ranging from 184 to $327 \mu \mathrm{m}$ ) interconnected pores. In vitro assays performed with osteoblastic cells showed no cytotoxicity associated with the synthesized nanocomposite. In vivo results after 29 days of implant showed that cells were able to penetrate through the porous structure to fully colonize the entire implant. Biocompatibility tests on polyurethane nanocomposites produced in this study revealed that this type of material can be potentially useful in tissue engineering of many different tissues, such as cartilage, bone, heart, valves, nerves, muscle, bladder, liver, among others. 


\section{Acknowledgements}

The authors would like to acknowledge the financial support from the following institutions: National Council for Scientific and Technological Development (CNPq), the State of Minas Gerais Research Foundation (FAPEMIG), and the National Synchrotron Light Laboratory (Brazil) for the use of their SAXS facilities.

\section{References}

1. Calandrelli L, Immirzi B, Malinconico M, Volpe MG, Oliva A and Ragione FD. Preparation and characterisation of composites based on biodegradable polymers for "in vivo" application. Polymer. 2000; 41(22):8027-8033

2. Al-Ahmad MW, Gutwald R, Lauer G, Hübner U and Schmelzeisen R. How to optimize seeding and culturing of human osteoblast-like cells on various. Biomaterials. 2002; 23(16):3319-3328.

3. ElAmin SF, Lu HH, Khan Y, Burems J, Mitchell J, Tuan RS et al. Extracellular matrix production by human osteoblasts cultured on biodegradable polymers applicable for tissue engineering. Biomaterials. 2003; 24(7):1213-1221.

4. Grad S, Kupcsik L, Gorna K, Gogolewski S and Alini M. The use of biodegradable polyurethane scaffolds for cartilage tissue engineering: potential and limitations. Biomaterials. 2003; 24(28):5163-5171.

5. Guan J, Fujimoto KL, Sacks MS and Wagner WR. Preparation and characterization of highly porous, biodegradable polyurethane scaffolds for soft tissue applications. Biomaterials. 2005; 26:3961-3971.

6. Guan J, Sacks MS, Beckman EJ and Wagner WR. Biodegradable poly(ester-urethane)urea elastomers based on poly(ether ester) triblock copolymers and putrescine: Synthesis, characterization, and cytocompatibility. Biomaterials. 2004; 25(18):85-96.

7. Williamson MR, Black R and Kielty C. PCL-PU composite vascular scaffold production for vascular tissue engineering: attachment, proliferation and bioactivity of human vascular endothelial cells. Biomaterials. 2006; 27(19):3608-3616.

8. Jung JH, Ree M and Kim H. Acid- and base-catalyzed hydrolyses of aliphatic polycarbonates and polyesters. Catalysis Today. 2006; 115(1-4):283-287.

9. Tay FR, Pashley DH, Williams MC, Raina R, Loushine RJ, Weller RN et al. Susceptibility of a polycaprolactone-based root canal filling material to degradation. I. alkaline hydrolysis. Endodontics. 2005; 31(8):593-598.

10. Cretu A, Gattin R, Brachais L and BarbierBaudry D. Synthesis and degradation of poly (2-hydroxyethyl methacrylate)-graft-poly (E-caprolactone) copolymers. Polymer Degradation and Stability. 2004; 83(3):399-404.

11. Hou Y, Chen J, Sun P, Gan Z and Zhang G. In situ investigations on enzymatic degradation of poly( $\varepsilon$-caprolactone). Polymer. 2007; 48(21):6348-6353.

12. Santerre JP, Woodhouse K, Laroche G and Labow RS. Understanding the biodegradation of polyurethanes: From classical implants to tissue engineering materials. Biomaterials. 2005; 26(35):7457-7470.

13. Howard GT. Biodegradation of polyurethane: a review. International Biodeterioration \& Biodegradation. 2002; 49:245-252.

14. Spaans CJ, Belgraver VW, Rienstra O, Groot JH, Veth RPH and Pennings AJ. Solvent-free fabrication of micro-porous polyurethane amide and polyurethane-urea scaffolds for repair and replacement of the knee-joint meniscus. Biomaterials. 2000; 21(23):2453-2460.

15. Pattanayak A and Jana SC. Thermoplastic polyurethane nanocomposites of reactive silicate clays: effects of soft segments on properties. Polymer. 2005; 46(14):5183-5193.
16. Lee JH, Park TG, Park HS, Lee DS, Lee YK, Yoon SC et al. Thermal and mechanical characteristics of poly(L-lactic acid) nanocomposite scaffold. Biomaterials. 2003; 24(16):2773-2778.

17. Savolainen K, Alenius H, Norppa H, Pylkkanen L, Tuomi T and Kasper G. Risk assessment of engineered nanomaterials and nanotechnologies-A review. Toxicology. 2010; 269(2-3):92-104.

18. Hussain SM, Hess KL, Gearhart JM, Geiss KT and Schlager JJ. In vitro toxicity of nanoparticles in BRL 3A rat liver cells. Toxicology in Vitro. 2005; 19(7):975-983.

19. Lin W, Huang $\mathrm{Y}$, Zhou XD and Ma Y. In vitro toxicity of silica nanoparticles in human lung cancer cells. Toxicology and Applied Pharmacology. 2006; 217(3):252-259.

20. Martinez-Gutierrez F, Olive PL, Banuelos A, Orrantia E, Nino N, Sanchez $\mathrm{EM}$ et al. Synthesis, characterization, and evaluation of antimicrobial and cytotoxic effect of silver and titanium nanoparticles. Nanomedicine: Nanotechnology, Biology and Medicine. 2010; in press.

21. Sadiq IM, Chowdhury B, Chandrasekaran $\mathrm{N}$ and Mukherjee A. Antimicrobial sensitivity of Escherichia coli to alumina nanoparticles. Nanomedicine: Nanotechnology, Biology and Medicine. 2009; 5(3):282-286

22. Grabinski C, Hussain S, Lafdi K, BraydichStolle L and Schlager J. Effect of particle dimension on biocompatibility of carbon nanomaterials. Carbon. 2007; 45(14):2828-2835.

23. SimonDeckers A, Gouget B, MayneL'Hermite M, HerlinBoime N, Reynaud $\mathrm{C}$ and Carriere M. In vitro investigation of oxide nanoparticle and carbon nanotube toxicity and intracellular accumulation in A549 human pneumocytes. Toxicology. 2008; 253(1-3):137-146

24. Lee YH, Kuo TF, Chen BY, Feng YK, Wen YR, Lin WC et al. Toxicity assessment of montmorillonite as a drug carrier for pharmaceutical applications: yeast and rats model. Biomedical Engineering: Applications, Basis and Communications. 2005; 17(2):72-78.

25. Choi SJ, Oh JM and Choy JH. Toxicological effects of inorganic nanoparticles on human lung cancer A549 cells. Journal of Inorganic Biochemistry. 2009; 103(3):463-471.

26. Styan KE, Martin DJ and PooleWarren LA. J. In vitro fibroblast response to polyurethane organosilicate nanocomposites. Journal Biomedical Materials Research A. 2008; 86(3):571-582.

27. Malec EJ and David DJ. Analytical chemistry of polyurethane. New York: Wiley; 1969.

28. Valério P, Guimarães MH, Pereira MM, Leite MF and Góes A. Primary osteoblast cell response to sol-gel derived bioactive glass foams. Journal of Materials Science: Materials in Medicine. 2005; 16(9):851-856.

29. Medrado SC, Machado CB, Valério P, Sanches MD and Góes A. The effect of a chitosan-gelatin matrix and dexamethasone on the behavior of rabbit mesenchymal stem cells. Biomedical Materials 2006; 1(3):155-161.

30. Lee HT and Lin LH. Waterborne Polyurethane/Clay Nanocomposites: Novel Effects of the Clay and Its Interlayer Ions on the Morphology and Physical and Electrical Properties. Macromolecules. 2006; 39(18):6133-6141.

31. Shor L, Güçeri S, Wen X, Gandhi M and Sun W. Fabrication of three-dimensional polycaprolactone/hydroxyapatite tissue scaffolds and osteoblast-scaffold interactions in vitro. Biomaterials. 2007; 28(35):5291-5297.

32. Anselme K. Osteoblast adhesion on biomaterials. Biomaterials. 2000; 21(7):667-681.

33. Turhani D, Weißenböck M, Watzinger E, Yerit K, Cvikl B, Ewers R et al. In vitro study of adherent mandibular osteoblast-like cells on carrier materials. International Journal of Oral \& Maxillofacial Surgery. 2005; 34(5):543-550. 